\title{
Performance analysis in esports: part 2 - modelling performance at the 2018 League of Legends World Championship
}

\author{
Andrew R. Novak ${ }^{1,2}$, Kyle J. M. Bennett3,4, Matthew A. Pluss ${ }^{1}$, and Job Fransen ${ }^{1}$
}

\begin{abstract}
${ }^{1}$ Human Performance Research Centre, Sport and Exercise Science, Faculty of Health, University of Technology Sydney, Moore Park, Australia.

${ }^{2}$ High Performance Department, Rugby Australia, Moore Park, Australia.

${ }_{3}^{3}$ School of Health and Human Sciences, Southern Cross University, Coffs Harbour, Australia.

${ }_{4}^{4}$ Centre for Athlete Development, Experience \& Performance, Southern Cross University, Coffs Harbour, Australia.
\end{abstract}

\begin{abstract}
Performance analysis is a well-established discipline in sports science, supported by decades of research. Comparatively, performance analysis in electronic sports (esports) is limited. Therefore, there is opportunity to accelerate performance outcomes in esports by applying methods grounded in sports science. The current study adopted a coach-centred approach to model performance at the 2018 League of Legends World Championship. Three expert coaches rated the proposed relationship between 43 variables and match outcomes in professional League of Legends competition using a Likert scale (1-10). The Likert scale was anchored with 'no relationship' at 1 and 'very strong relationship' at 10. The coaches' median ratings were calculated for each variable. Variables with a median score $\geq 6$ were retained for analyses. A total of 14 variables were collected from the 2018 League of Legends World Championship $(n=119)$ matches via video annotations and match histories. Generalized Linear Mixed Effects Models with binomial logit link function were implemented with respect to the Blue Side winning or losing the match, and individual teams were specified as random effects. Variables were screened for multicollinearity before a step-up approach was utilised. The best model of performance included Tower Percentage $(\mathrm{p}=0.006)$ and Number of Inhibitors ( $\mathrm{p}=0.029)$. This model achieved a classification accuracy of $95.8 \%$. While Tower Percentage and Number of Inhibitors contributed to winning or losing, further research is required to determine effective strategies to improve these variables, to understand the relevance of these variables across the complete time-series of the match, and to determine whether performance indicators remain stable across game updates.
\end{abstract}

\section{Key Words}

Esports, Performance, Notational Analysis, Video Games, Technology.

\section{DOI}

http://dx.doi.org/10.31236/osf.io/84fmy

\section{Citation}

Novak, A. R., Bennett, K. J. M., Pluss, M. A., \& Fransen, J. (2019). Performance analysis in esports: part 2 - modelling performance at the 2018 League of Legends World Championship. SportRxiv. doi: 10.31236/osf.io/84fmy 


\section{INTRODUCTION}

Electronic sports (esports) is a modern competitive environment where players compete against each other via human-computer interactions (Pluss et al., 2019). There are numerous genres of games within esports, and a classification of Action Video Games has emerged that includes Multiplayer Online Battle Arenas, First Person Shooters, Third Person Shooters and Real-time Strategy (Kowal, Toth, Exton, \& Campbell, 2018). One of the most widely played Action Video Games is League of Legends - a Multiplayer Online Battle Arena game which was first released in 2009, with up to 100 million active users recorded per month (Statista, 2016). In League of Legends, two teams of five players work against each other to destroy the opposing team's Nexus (i.e. the main structure located at the opposite side of the arena). The arena consists of three lanes that cross from the bottom left corner (Blue Side) to the top right corner (Red Side), with jungle areas separating each lane and a river passing from the top left to bottom right (Figure 1). At the start of the game, players select from a pool of 145 champions (the current champion pool size as of September, 2019) that they will control as their in-game avatar throughout each match, each of which has unique abilities and skills that the player can activate. As players eliminate opposing Champions, waves of enemy Minions, and various other objectives (e.g. enemy Towers and Inhibitors), their Champion become more powerful and gains new abilities, allowing them to more easily overcome their opponents and ultimately win the match (Novak, Bennett, Pluss, \& Fransen, [Preprint]). 


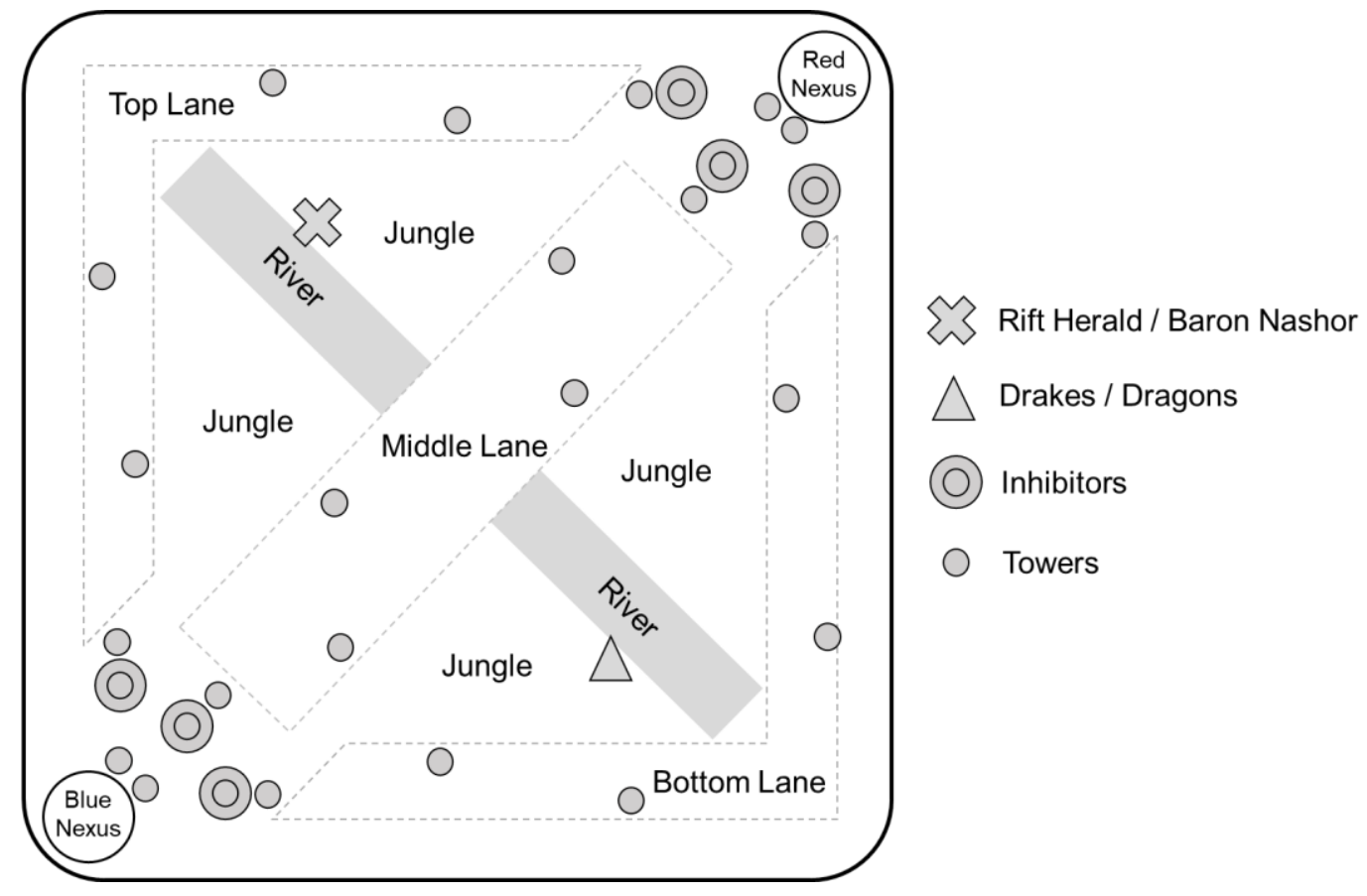

Figure 1: League of Legends arena

League of Legends is currently played competitively across numerous regions (Korea, North America, Europe West, Europe Nordic and East, Oceania, Russia, Turkey, Brazil, Latin America North, Latin America South, Vietnam, South-East Asia, and Japan). The number of ranked players varies between 200,000 players in Oceania and Russia, to more than 3.5 million ranked players in Korea (data accessed via website https://[region].op.gg/statistics/tier, 15/09/2019)). Many players are employed as professionals on full-time contracts and the 2018 League of Legends World Championship boasted a prize pool of $\$ 6$ million USD (retrieved 24/09/2019 from https://www.esportsearnings.com/tournaments).

To date, most of the video gaming research has focused on the relationship between participation and domain general perceptual-cognitive and perceptual-motor skills. For example, there is some evidence that video gaming is associated with enhanced working 
memory capacity and information processing skills (Colzato, van den Wildenberg, Zmigrod, \& Hommel, 2013; Powers, Brooks, Aldrich, Palladino \& Alfieri, 2013). Furthermore, it is suggested that expertise in multiplayer online battle arena games is related to domain general cognitive skills and numerical processing (Bonny \& Castaneda, 2017). However, a metaanalysis of experimental studies yielded negligible effects of video gaming on executive functions such as working memory, multitasking, nonverbal intelligence, and task switching (Powers et al., 2013). While there is conflicting evidence concerning the relationship between domain general skills and video gaming, no research has investigated the in-game actions associated with successful match outcomes within professional esports competitions (i.e. competitive video gaming).

Due to the lack of available peer-reviewed scientific research, professional esports coaches and players often rely on unvalidated websites and statistics, individual experiences and anecdotes (Novak et al., [Preprint]). Alternatively, coaches and players may apply research from domains that exhibit some similar characteristics to esports such as Chess (Bonny \& Castaneda, 2016; Pluss et al., 2019). In an effort to improve the quality of evidence available to esports coaches and practitioners, we recently discussed how the field of performance analysis in sport (Hughes, Barnes, Churchill \& Stone, 2017; Hughes \& Bartlett, 2002; Hughes \& Franks, 2004; McGarry, 2009), could be adopted by those concerned with esports performance (Novak et al., [Preprint]). However, while esports games often make data available on publicly accessible websites, little is known about the validity of these data which are captured, aggregated, and visualised via proprietary software systems. Therefore, we investigated the validity of automated Match History statistics in professional League of Legends competition by drawing on methodologies grounded in traditional sports science (Hughes et al., 2017; Robertson et al., 2016; Vaz et al., 2010). We found that we could validly capture match statistics by using match histories and video annotation, except for several 
variables such as Wards Placed and Wards Destroyed. The ability to validly capture data via match histories and video annotation is important, particularly when one of the two data sources does not populate with data, as was observed in several cases for professional matches. However, while the data collected by these methods may be valid, it is not yet known which of these available variables are associated with performance outcomes. Therefore, this study is the first adopt a coach-centred approach to model performance at the 2018 League of Legends World Championship using a variety of in-game match statistics.

\section{MATERIALS AND METHODS}

\subsection{Coach analysis of performance variables}

A survey was created by three of this study's authors, who each had 255, 1596 and 1401 hours experience playing League of Legends (data extracted from user accounts via website: https://wol.gg/ data extracted 03/08/2019), as well as many hours viewing professional competitions and two years' experience consulting with professional League of Legends teams and coaches. Variables were included based on the collective agreement of these players via open discussion, and if the variables could be collected from Match Histories and/or video annotation as per Novak et al. (Preprint). The final survey identified 43 potential performance variables and posed the question "select how strong you believe the variable is related to match outcome in professional League of Legends" for each variable. A Likert scale was utilised with ratings between 1-10 (no middle option available), and with only two descriptors providing context as anchors at each end of the scale $(1=$ "no relationship", and $10=$ "very strong relationship"). A final question asked coaches to list any additional performance indicators that had not been included within the survey. The survey was developed using Google Forms and was sent via email to League of Legends coaches and the complete list of performance indicators is listed in Table 1 . Three coaches $(n=3)$ responded to the survey. 


\subsection{League of Legends World Championship}

The 2018 World Championship was comprised of 24 teams from 14 regions (Table 2). The game version was v8.19. Due to historical performance variations across regions, top performing regions were permitted to each enter their best three teams, while lower performing regions each entered their single best team (the winning team at yearly regional finals). A total of 119 matches were played throughout the World Championship, with the early stages of the tournament following a round-robin format, while knockout stages such as the finals followed a best-of-five format. Due to these factors, regions and teams did not play an equal number of games (average $=10 \pm 6$ games per team, Table 1 ). Given that draws are not possible in League of Legends, no matches needed to be excluded from analysis when examining win/loss outcomes.

\subsection{Performance indicators}

Video footage for the 2018 League of Legends World Championship was accessed and viewed via the public League of Legends Video On Demand repository (https://watch.lolesports.com/vods), while Match Histories were accessed via the public League of Legends Match History repository (https://matchhistory.na.leagueoflegends.com). For each match, the region and name of the winning and losing team were recorded, as well as the team sides (Blue vs. Red) and match duration. Performance indicators determined via the coach survey were recorded within three categories: 1) frequency data (e.g. number of kills, deaths and assists for each player); 2) time-dependent data (e.g. the exact time that the first tower was destroyed); and 3) categorical data (e.g. which team destroyed the first tower). Table 1 displays the complete list of variables. Institutional ethics approval was received prior to undertaking this study. 


\subsection{Statistical analysis}

\subsubsection{Coach survey}

Data from the coaching survey were exported from Google Forms as a spreadsheet. For each variable, the Median value of the three coaches' responses was calculated using Microsoft Excel. Variables with a Median value $\geq 6$ were retained in the final analysis of 2018 League of Legends world championship.

\subsubsection{League of Legends World Championship}

Variables retained after the coach survey were manually inputted into a spreadsheet for each match by one of the experienced players/authors via manually copying data from the Match Histories or video annotation from the Video on Demand. Variables that contextualised the frequencies (e.g. own team gold vs opposition gold) were expressed as percentages of total match values (e.g. own team gold / [own team gold + opposition gold]) for modelling purposes as per previous recommendations for variable normalisation in performance analysis (Hughes \& Franks, 2004). Analyses were conducted using the R statistical framework (R Development Core Team, 2010). Variables were initially examined for multicollinearity using a correlation matrix. If a correlation of $\geq 0.8$ was observed between two variables, only the variable with the greatest correlation with respect to match outcome was retained for analysis. Given that there was an uneven distribution of matches played by each team, generalized linear mixed effects models were deemed appropriate to examine the relationships between performance indicators and match outcomes, as some correlations within groups were expected. The "glmer" function within the "lme4" package was used (v1.1-21; Bates et al., 2019) and the Blue Side Team Name was specified as a random effect, while all other independent variables were specified as fixed effects. All matches were analysed with respect to the Blue side, and a binomial link function was adopted to analyse the data with respect to the Blue side winning or losing the match. While all four measures of Vision were retained after the coach survey, 
our previous data quality concerns regarding variables relating to vision (e.g. Wards Placed and Wards Destroyed; Novak et al., [Preprint]), resulted in a decision to remove those four variables. Therefore, 24 variables remained for further analysis. The 24 variables were assessed for Multicollinearity, and a further 10 variables were removed, leaving a final 14 variables. Given that there were 14 variables remaining and only 119 observations, a step-up approach was adopted when modelling the generalized linear mixed effects models. In this process, a null model was firstly specified. Subsequently, each variable was added one-by-one as a fixed effect to identify the variable that explained the most variance by comparing the new model against the null model via the anova function in $\mathrm{R}$ to view the alpha value and Akaike Information Criterion (AIC). Models were also assessed by comparing the predicted vs. observed values to calculate the classification accuracy of the model. The residuals for each model were also examined via QQ plots to assess normality. Following the identification of the best model with one fixed effect, a second fixed effect was added one-by-one as per the first model and assessed via the same method until no further model improvement was observed.

\section{RESULTS}

\subsection{Coach Survey}

Three coaches completed the survey. Two were head coaches and one was an assistant coach at the time of data collection, each with 3-4 years' experience coaching, and 7-9 years playing League of Legends. Two coaches had coached at the highest level in their region (Professional) and one had coached at the second highest level (Academy). Of the 43 variables analysed, 28 were identified with a Median score $\geq 6$, and therefore retained for subsequent analysis. These variables are represented in Table 1 and are related to Champion Power, Objectives, and Vision. No variables relating to Kills, Deaths and Assists were given a Median score $\geq 6$ by the three coaches. Only one coach responded to the question asking them to identify any other potential performance indicators. This coach noted that "gold lead in 
Performance analysis in esports: part 2 - modelling performance at the 2018 League of Legends World Championship

relation to the time in the game" would be important and would be rated 9/10 on the Likert Scale. However, given that time-series analysis was not within the scope of this study and that the other two coaches did not include a similar comment, this variable was not included. 
Performance analysis in esports: part 2 - modelling performance at the 2018 League of Legends World Championship

Table 1: Variables include in coach survey

\begin{tabular}{|c|c|c|c|c|}
\hline Category & Performance Indicator & $\begin{array}{c}\text { Median } \\
\text { Coach } \\
\text { Score } \\
\end{array}$ & $\begin{array}{l}\text { Retained } \\
\text { After } \\
\text { Coach } \\
\text { Survey } \\
\end{array}$ & $\begin{array}{c}\begin{array}{c}\text { Retained } \\
\text { after } \\
\text { multicollinearity } \\
\text { assessment }\end{array} \\
\end{array}$ \\
\hline \multirow{8}{*}{$\begin{array}{l}\text { Champion } \\
\text { Power }\end{array}$} & Champion Level & 7 & Yes & No \\
\hline & Creep Ratio (own creeps vs opposition creeps) & 6 & Yes & Yes \\
\hline & Creep Score & 6 & Yes & No \\
\hline & Creeps per Minute & 7 & Yes & Yes \\
\hline & Gold per Minute & 6 & Yes & No \\
\hline & Gold Ratio (own gold vs opposition gold) & 6 & Yes & Yes \\
\hline & Level Ratio (own level vs opposition level) & 6 & Yes & No \\
\hline & Total Gold & 6 & Yes & Yes \\
\hline \multirow{14}{*}{$\begin{array}{l}\text { Kills, } \\
\text { Deaths \& } \\
\text { Assists }\end{array}$} & Assist Ratio (own assists vs opposition assists) & 1 & No & No \\
\hline & Death by First Blood & 1 & No & No \\
\hline & Death Ratio (own deaths vs opposition deaths) & 4 & No & No \\
\hline & First Blood & 3 & No & No \\
\hline & First Blood Assist & 2 & No & No \\
\hline & KDA (Kills + Assists)/Deaths & 1 & No & No \\
\hline & KDA Ratio (Own KDA vs opposition KDA) & 1 & No & No \\
\hline & Kill Ratio (own kills vs opposition kills) & 5 & No & No \\
\hline & Number of Assists & 2 & No & No \\
\hline & Number of Assists per Minute & 1 & No & No \\
\hline & Number of Deaths & 4 & No & No \\
\hline & Number of Deaths per Minute & 1 & No & No \\
\hline & Number of Kills & 2 & No & No \\
\hline & Number of Kills per Minute & 1 & No & No \\
\hline \multirow{17}{*}{ Objectives } & Baron Ratio (own Barons vs opposition Barons) & 9 & Yes & No \\
\hline & Dragon Ratio (own Dragons vs opposition Dragons) & 6 & Yes & No \\
\hline & First Baron & 9 & Yes & Yes \\
\hline & First Dragon & 5 & No & No \\
\hline & First Elder Dragon & 8 & Yes & No \\
\hline & First Inhibitor & 8 & Yes & No \\
\hline & First Tower & 7 & Yes & Yes \\
\hline & Number of Cloud Drakes & 6 & Yes & Yes \\
\hline & Number of Elder Dragons & 6 & Yes & Yes \\
\hline & Number of Elemental Drakes & 7 & Yes & Yes \\
\hline & Number of Infernal Drakes & 7 & Yes & Yes \\
\hline & Number of Inhibitors & 8 & Yes & Yes \\
\hline & Number of Mountain Drakes & 8 & Yes & Yes \\
\hline & Number of Ocean Drakes & 6 & Yes & Yes \\
\hline & Number of Towers & 10 & Yes & No \\
\hline & Rift Herald & 8 & Yes & Yes \\
\hline & Tower Ratio (own towers vs opposition towers) & 9 & Yes & No \\
\hline \multirow{4}{*}{ Vision } & Vision Ratio (own vision vs opposition vision) & 7 & Yes & No * \\
\hline & Vision Score & 7 & Yes & No * \\
\hline & Wards Destroyed & 8 & Yes & No * \\
\hline & Wards Placed & 6 & Yes & No * \\
\hline
\end{tabular}

Note: * denotes variable that coaches identified for inclusion but were excluded due to previous data quality concerns (Novak et al., [preprint]). 
Table 2: Number of games played per region and team during the 2018 League of Legends World Championship

\begin{tabular}{|c|c|c|c|}
\hline Region & $\begin{array}{c}\text { Games } \\
\text { per region }\end{array}$ & $\begin{array}{l}\text { Number } \\
\text { of teams }\end{array}$ & $\begin{array}{c}\text { Games } \\
\text { per team }\end{array}$ \\
\hline Brazil & 5 & 1 & 5 \\
\hline \multirow[t]{3}{*}{ China } & 47 & 3 & 12 \\
\hline & & & 17 \\
\hline & & & 18 \\
\hline Commonwealth of Independent States & 9 & 1 & 9 \\
\hline \multirow[t]{3}{*}{ Europe } & 47 & 3 & 6 \\
\hline & & & 17 \\
\hline & & & 24 \\
\hline Japan & 8 & 1 & 8 \\
\hline \multirow[t]{3}{*}{ Korea } & 26 & 3 & 6 \\
\hline & & & 9 \\
\hline & & & 11 \\
\hline Latin America North & 8 & 1 & 8 \\
\hline Latin America South & 4 & 1 & 4 \\
\hline \multirow[t]{3}{*}{ North America } & 34 & 3 & 6 \\
\hline & & & 6 \\
\hline & & & 22 \\
\hline Oceania & 4 & 1 & 4 \\
\hline South-East Asia & 4 & 1 & 4 \\
\hline \multirow[t]{3}{*}{ Taiwan, Hong Kong and Macao } & 27 & 3 & 6 \\
\hline & & & 7 \\
\hline & & & 14 \\
\hline Turkey & 9 & 1 & 9 \\
\hline Vietnam & 6 & 1 & 6 \\
\hline
\end{tabular}

\subsection{League of Legends World Championship}

Of the 119 matches, the Blue side won 65 and Red side won 54. Additional descriptive statistics can be found in Table 3 as mean \pm SD. During the assessment of multicollinearity, Tower Percentage was the strongest variable, resulting in the removal of seven variables that were multicollinear with Tower Percentage (Level Percentage, Towers Taken, Inhibitor Percentage, First Inhibitor, Barons, Gold Percentage, and Gold Per Minute). Additionally, Tower Percentage was the strongest variable in the generalized linear mixed effects model, becoming the variable retained at the first step of the step-up approach. Subsequently, the 
number of Inhibitors Taken was retained in the model at the second step of the step-up approach and the inclusion of additional variables thereafter did not improve the model any further. The final model that included Tower Percentage and number of Inhibitors Taken as fixed effects was significantly better than the null model $(\mathrm{p}<0.001)$, reducing the AIC from 148.8 to 37.4 and achieving a prediction accuracy of $95.8 \%$ when comparing the predicted values to the observed values for match outcome. Specifications of the final model are presented in Table 4 and estimates have been exponentiated to allow for interpretations as odds ratios.

Table 3: Descriptive team-based statistics of the 2018 League of Legends World Championship

\begin{tabular}{|c|c|c|}
\hline & Blue Side & Red Side \\
\hline Match Duration (min) & \multicolumn{2}{|c|}{$32.27 \pm 5.96$} \\
\hline Win Rate (\%) & 54.62 & $45 \cdot 38$ \\
\hline Rift Herald (\%) * & 61.34 & 35.29 \\
\hline First Tower (\%) & 57.63 & 42.37 \\
\hline First Baron (\%) * & 50.42 & 44.54 \\
\hline Level & $75.43 \pm 7.58$ & $74.54 \pm 8.43$ \\
\hline Level Percentage & $50.33 \pm 2.31$ & $49.67 \pm 2.31$ \\
\hline Creep Score & $1089 \pm 207$ & $1083 \pm 227$ \\
\hline Creep Score Per Minute & $33.82 \pm 2.91$ & $33.48 \pm 2.53$ \\
\hline Creep Score Percentage & $50.23 \pm 2.73$ & $49.77 \pm 2.73$ \\
\hline Towers Taken & $6.38 \pm 3.81$ & $5.20 \pm 3.92$ \\
\hline Tower Percentage & $55.63 \pm 34.26$ & $44.37 \pm 34.26$ \\
\hline Inhibitors Taken & $0.91 \pm 0.95$ & $0.60 \pm 0.83$ \\
\hline Dragons Taken & $1.42 \pm 1.1$ & $1.66 \pm 1.21$ \\
\hline Dragon Percentage & $47.2 \pm 35.99$ & $52.8 \pm 35.99$ \\
\hline Elder Dragons Taken & $0.03 \pm 0.16$ & $0.08 \pm 0.3$ \\
\hline Barons Taken & $0.64 \pm 0.66$ & $0.56 \pm 0.67$ \\
\hline Gold (In Thousands) & $57.67 \pm 11.46$ & $56.14 \pm 13.00$ \\
\hline Gold Per Minute & $1795 \pm 221$ & $1734 \pm 213$ \\
\hline Gold Percentage & $50.85 \pm 5.79$ & $49.15 \pm 5.79$ \\
\hline
\end{tabular}

Note: all variables are calculated as a sum of the five individual team players. ${ }^{*}=$ variable was not obtained in all games i.e. values for blue and red side do not add to $100 \%$ 
Performance analysis in esports: part 2 - modelling performance at the 2018 League of Legends World Championship

Table 4: Best model of performance for the 2018 League of Legends World Championship

\begin{tabular}{lccccccc}
\hline & Estimate & $\begin{array}{c}\mathbf{9 5 \%} \text { CI } \\
\text { lower }\end{array}$ & $\begin{array}{c}\text { 95\% CI } \\
\text { upper }\end{array}$ & $\begin{array}{c}\text { Standard } \\
\text { Error }\end{array}$ & z value & p & $\begin{array}{c}\text { Odds } \\
\text { Ratio }\end{array}$ \\
\hline Intercept & -6.674 & -14.825 & -3.866 & 1.830 & -3.646 & $<0.001$ & 0.001 \\
Tower Percentage & 0.084 & 0.032 & 0.186 & 0.031 & 2.738 & 0.006 & 1.087 \\
Inhibitors Taken & 2.568 & 0.681 & 7.616 & 1.179 & 2.178 & 0.029 & 13.036 \\
\hline
\end{tabular}

\section{DISCUSSION}

The current study is the first to undertake an analysis of in-game performance indicators within a professional esports tournament (i.e. the 2018 League of Legends World Championship). The aim of the study was to apply methods grounded in sports science to model esports performance. This was achieved via engaging with expert coaches, and performance analysis facilitated by implementation of video annotation and validated match statistics. This study utilised generalized linear mixed effects models and identified that the percentage of Towers Taken was most strongly related to match outcome, while the number of Inhibitors Taken also contributed to the best model of performance (95.8\% classification accuracy). Specifically, an improvement of $1 \%$ in Tower Percentage resulted in $8.7 \%$ greater odds of winning the match, while with each additional Inhibitor Taken, there was a thirteenfold increase in the odds of winning the match.

This study is the first to provide evidence for the importance of Towers and Inhibitors for match outcomes in League of Legends. However, neither of these findings were surprising, given that Towers are the main structures that prevent players from progressing towards the opponent's Nexus. Towers cause large amounts of damage to players, especially during the early phases of the game when Champions have limited defences and relatively low health. They also prevent access to key objectives such as the opponent's Inhibitors and the Nexus. Additionally, Inhibitors provide an important effect that helps teams to end the game 
(stronger Minion waves are deployed towards the opponent's Nexus to help the team destroy it). While Towers and Inhibitors are evidently important for successful performance, further research is required to determine effective strategies to improve Tower Percentage and destroy Inhibitors. Specifically, to improve Tower Percentage, teams need to destroy opposing Towers while simultaneously protecting their own Towers. Additionally, given that there are 11 Towers on each side, there may be optimal strategies for targeting Towers at specific moments of the game, which should be explored in future research.

Interestingly, the First Tower objective did not contribute to the model of performance. Further research should also determine whether Towers at various locations are of greater importance than others. It should be noted that two of the Towers are located next to the Nexus and are often destroyed during the final moments of the game. Therefore, it is possible that the Tower Percentage is somewhat artificially inflated as a measure of performance in the final statistics, given that the game state in which the winning team finally overcame their opponents may have occurred during a final team fight i.e. prior to taking these final two Towers. Time-series analysis was beyond the scope of this study but is suggested for further investigation. These findings based on the objective data are in support of the coaches' subjective opinions, who also indicated that number of Towers Taken is the most important variable (the only variable with a median value of 10/10), while they also rated Tower Ratio as $9 / 10$.

Given the previous study (Novak et al., [Preprint]), which observed 15 matches of the North American League of Legends Championship Series (NA LCS) and 15 matches of the League of Legends European Championship (EU LEC), some comparisons can be made between these regions and the World Championship competition. Notably, the Blue side won a greater percentage of matches in all three competitions, while match duration was 
approximately the same (32 $\pm 6 \mathrm{~min})$ across the three data sets. Interestingly, while the number of Inhibitors Taken was significant in the performance model, this variable appears slightly lower at World Championship (mean $=0.91$ and 0.60 for Blue and Red sides respectively), compared with NA LCS (1.6 Blue side and 0.73 Red side) and EU LEC (1.1 Blue side and 0.9 Red side). It should be noted that this study utilised an unbalanced data set due to the format of the League of Legends World Championship i.e. teams and regions were represented by vastly different numbers of matches, ranging between 4 and 24 for individual teams, and between 4 and 47 for regions (Table 1). Therefore, the summary statistics (mean \pm SD; Table 2) may be skewed towards game styles of those teams with greater representation. However, further research can be used to determine whether differences in play styles exist between regions. Collectively, there are many avenues for future research given the current lack of published evidence.

\section{LIMITATIONS}

This research should be interpreted considering several limitations. First, it is noted that only three coaches responded to the survey, and therefore, the survey results cannot be generalised. Future research would benefit by seeking engagement from a greater number of industry experts. However, the findings of this study supported the opinions of the three coaches. Second, due to the inclusion bias towards previously high-performing regions (e.g. China, Europe, North America and Korea) and the knock-out style format of competition, there may be inherent bias within the data towards teams who played more games (i.e. the results may reflect specific gameplay styles from China and Europe who each played 47 games). While this was accounted for within the mixed effects model, it could not be accounted for within the summary statistics. Given that the League of Legends World Championship encompasses the top teams across all competitive global regions, it is accepted that the summary statistics encompass current performances at the highest level of competitive League 
of Legends. Third, it should be noted that League of Legends receives relatively minor updates roughly every two weeks (e.g. minor changes to champion skills, cooldowns, or adding new champions to the game), while larger updates typically occur once per calendar year, (e.g. changes to the way the Rune system works [abilities that players select prior to each match] or adding new defences to the Towers to change how players strategize). As noted in this study, the patch version for the 2018 World Championships was v8.19 and future research should be conducted to determine performance indicators that are longitudinally related to successful performance. Finally, due to the limited sample size, it was not feasible to include many independent variables or to withhold a sample to facilitate predictions on unseen data. Future research should aim to acquire larger samples so that more variables can be included, and the predictive power of the models can be assessed. This could be facilitated via the Riot Games Application Program Interface (API); however, validation of the API data quality is required prior to implementation.

\section{CONCLUSION}

The current study applied traditional performance analysis methods that were learned in the sports science domain, to help understand esports performance. At the highest level of League of Legends competition, Tower Percentage and Number of Inhibitors were identified as having the strongest relationships with performance. Therefore, further research should seek to identify strategies to effectively target these objectives across the time-series of the match, and to determine whether there are differences between World Championship competition and other competitions e.g. Professional level and Academy level within individual regions. 


\section{REFERENCES}

Bonny, J. W., \& Castaneda, L. M. (2017). Impact of the arrangement of game information on recall performance of Multiplayer Online Battle Arena players. Applied Cognitive Psychology 30(5): 664-671. DOI: 10.1002/acp.3234

Bonny, J. W., \& Castaneda, L. M. (2017). Number processing ability is connected to longitudinal changes in multiplayer online battle arena skill. Computers in Human Behavior 66: 377-387. DOI: 10.1016/j.chb.2016.10.005

Colzato, L., van den Wildenberg, W., Zmigrod, S. \& Hommel, B. (2013). Action video gaming and cognitive control: playing first person shooter games is associated with improvement in working memory but not action inhibition. Psychological Research 77(2): 234-239. DOI: 10.1007/s00426-012-0415-2

Hughes, A., Barnes, S., Churchill, S. M., \& Stone, J. A. (2017). Performance indicators that discriminate winning and losing in elite men's and women's Rugby Union. International Journal of Performance Analysis in Sport 17(4): 534-544. DOI: 10.1080/24748668.2017.1366759

Hughes, M. D., \& Bartlett, R. M. (2002). The use of performance indicators in performance analysis. Journal of Sports Sciences 20(10): 739-754. DOI: 10.1080/026404102320675602

Hughes, M \& Franks, I. (2004). Notational analysis of sport ( $2^{\text {nd }}$ ed.). London, England: Routledge.

McGarry, T. (2009). Applied and theoretical perspectives of performance analysis in sport: Scientific issues and challenges. International Journal of Performance Analysis in Sport 9(1): 128-140. DOI: 10.1080/24748668.2009.11868469.

Novak, A. R., Bennett, K. J. M., Pluss, M. A., \& Fransen, J. (Preprint). Performance analysis in esports: Part 1 - the validity and reliability of match statistics and notational analysis in League of Legends. SportRxiv DOI: 10.31236/osf.io/sm3nj

Pluss, M. A., Bennett, K. J. M., Novak, A. R., Panchuk, D., Coutts, A. J., \& Fransen, J. (2019). Esports: the chess of the $21^{\text {st }}$ century. Frontiers In Psychology. 1O(156):1-5. Doi: 10.3389/fpsyg.2019.00156

Powers, K., Brooks, P., Aldrich, N., Palladino, M. A., \& Alfieri, L. (2013). Effects of video-game play on information processing: A meta-analytic investigation. Pychonomic Bulletin \& Review 2O(6): 1055-1079. DOI: 10.3758/s13423-013-0418-z

Robertson, S., Gupta, R., \& McIntosh, S. (2016). A method to assess the influence of individual player performance distribution on match outcome in team sports. Journal of Sports Sciences 34(19): 1893-1900. DOI: 10.1080/02640414.2016.1142106

Statista. (2016). Number of League of Legends monthly active users (MAU) from 2011 to 2016. Retrieved 09/10/2019, from https://www.statista.com/statistics/317099/number-lolregistered-users-worldwide/

Vaz, L., Rooyen, M., \& Sampaio, J. (2010). Rugby game-related statistics that discriminate between winning and losing teams in IRB and Super Twelve close games. Journal of Sports Science \& Medicine 9(1): 51-55. 